\title{
Twists and turns on the way to progress in metastatic castrate-resistant prostate cancer
}

\author{
Oliver Sartor \\ Department of Medicine and Urology, Tulane University, New Orleans, Louisiana 70115, USA \\ Asian Journal of Andrology (2010) 12: 790-792. doi: 10.1038/aja.2010.113; publshed online 20 September 2010.
}

Harnessing the body's immune system for the treatment of metastatic cancer has been a dream since the late 19 th century [1]. Since that time progress has been intermittent and mostly disappointing. Interferon and Interleukin-2 represented steps forward but in retrospect the steps were not large and rarely are these agents used in the clinic today except as the control arm of randomized studies designed to demonstrate that a new drug is better.

Sipuleucel-T represents an interesting and important conceptual step forward. Books have been written about drug development but this one did not follow the script (in fact, a new book is probably justified). Yes, scientific advances translated into new therapeutic advance but the twists and turns along the way contained multiple unanticipated events. Further, the approval of this agent has led to fundamental questions about our society and what we value.

Correspondence to: Prof. Oliver Sartor

Fax: +1-504-988-5059

E-mail: osartor@tulane.edu
If the primary endpoint is not survival, but there is a survival benefit observed, how much does that matter? How large should a randomized trial be to justify approval? Which experts should evaluate new cancer therapies and comment on their suitability for approval? Who has the responsibility of protecting men and women who make regulatory decisions that are unpopular? How do we measure conflicts of interests and exclude those with real or apparent conflicts from the regulatory process? How much correlative science is required to create a sense of trust in clinical results? Should response rates and progression rates correlate with survival? Did the control group do worse than expected? How much can (or should) our society pay for incremental progress? Are we now reaching limits in terms of what we can afford to pay for new therapies? What are the ramifications and consequences if our society decides that some drugs are too expensive to provide? Although the FDA's ability to mandate post-marketing trials is unquestioned, who should be authorized to question the wisdom of their trial designs? Is this drug development or drama? Actually it is a touch of both. Some of these questions I will touch on and some I will not. For now, space constraints require only a handful of these issues be addressed in relationship to sipuleucel-T.

Let us start with the science and how scientific understanding led to a new medicine. Dranoff and colleagues demonstrated [2] in 1993 that granulocyte-monocyte colony stimulating factor (GM-CSF), when expressed in tumor cells, can inhibit cancerous growth in certain model systems. This fundamentally important observation led to experiments that combined GM-CSF and prostatic acid phosphatase to form a fusion protein that in turn is used to generate immune responses. This concept was scaled-up and applied to humans with prostate cancer. Large clinical trials have now led to a new FDA approval in the USA. This approval, in March 2010, was the first FDA approval for advanced prostate cancer since 2004. Sipuleucel-T was only the second agent in history to be associated with a survival benefit for metastatic castrate-resistant prostate cancer (mCRPC). 
Randomized trials in patients with mCRPC patients with minimal or no symptoms demonstrated a 4.1 month survival benefit for sipuleucel-T over 'control' treated patients. This large randomized trial (IMPACT or D9902B) with 512 patients was recently published in the New England Journal of Medicine [3]. The trial confirmed the survival benefit observed in smaller earlier trials (D9901) and served as an appropriate basis for the FDA approval.

Is the survival advantage in the IMPACT trial due to a poorly performing control group? No, survival in the control arm for IMPACT (21.7 months) was nearly identical to that previously observed [4] for asymptomatic $\mathrm{mCRPC}$ patients treated in the control group of the large atrasentan trial (20.3 months).

In IMPACT, no response rate or progression-free survival (PFS) advantage was seen for sipuleucel$\mathrm{T}$ over the control group. Is our current process of measuring response and progression truly flawed, or do post-progression alterations in tumor growth kinetics account for the interesting survival advantages observed? Clearly postprogression delays in growth are a possible explanation but this remains a hypothesis rather than fact (at this time). Does every trial with this class of agents now depend on analysis of overall survival (OS)? If both responses and PFS are unreliably measured, are there any other intermediate endpoints that can serve as an index of sipuleucel-T activity? The conundrum of PFS and survival being delinked is now becoming a familiar story in mCRPC. In a large randomized phase III trial, satraplatin/ prednisone had a PFS but not OS advantage over prednisone alone [5]. Similar findings with regard to PFS and OS were reported for bevacizumab/docetaxel/prednisone as compared to docetaxel/prednisone [6]. Randomized phase II studies with OGX-011 [7] and ZD4054 [8] have been associated with OS but not PFS improvements. Have we been deluding ourselves for years when evaluating PFS, or are the new agents different to the old ones? There is clearly more we need to understand about markers of progression in $\mathrm{mCRPC}$.

Now that survival advantages with sipuleucel-T are demonstrated, the FDA has approved the drug, and patients are clamoring for this novel treatment that has a bit of panache; what is the next step? Ordinarily the next step would focus on new indications (such as earlier stage prostate cancer), or new therapeutic combinations (e.g. ipilumimab plus sipuleucel-T comes to mind). However this usual discussion has been subverted by the price of therapy. The sponsor (Dendreon) has decreed that centers administering the drug will be billed $\$ 93000$ for the three infusions required for therapy. Costs to the patient and their insurance companies will be considerably more after administration and other costs are calculated. How is such a price justified? For that matter how is the cost of any new drug in the USA determined and justified? Do we need to re-think the current process and focus discussion on why medical care in the USA consumes a greater percentage of the gross domestic product (GDP) as compared to any other nation? Is now the time to do so?

The debate on costs involves many stakeholders and the con- sequences are large. Investment in new products is fueled by the expectation of profitable returns and if poor returns on investment become an expectation, then new progress against dreaded disease will not be forthcoming. The USA has marvelous medical technology and breath-taking costs but the lifespan of our citizens is not longer than our peers. How this debate is resolved will be key to the future of both medicine and the global economy.

\section{References}

1 Coley WB. The treatment of malignant tumors by repeated inoculations of Erysipelas, with a report of ten original cases. Am J Med Sci 1893; 105: 487-511.

2 Dranoff G, Jaffee E, Lazenby A, Golumbek P, Levitsky H, et al. Vaccination with irradiated tumor cells engineered to secrete murine granulocyte-macrophage colonystimulating factor stimulates potent, specific, and long-lasting anti-tumor immunity (tumor immunology/gene transfer). Proc Natl Acad Sci USA 1993: 90; 3539-43.

3 Kantoff PW, Higano CS, Shore ND, E. Berger R, Small EJ, et al. Sipuleucel-T immunotherapy for castration-resistant prostate cancer for the IMPACT study investigators. N Engl J Med 2010; 363: 411-22.

4 Carducci MA, Saad F, Abrahamsson PA. Phase 3 randomized controlled trial of the efficacy and safety of atrasentan in men with metastatic hormone-refractory prostate cancer. Cancer 2007; 110: 1959-66.

5 Sternberg CN, Petrylak DP, Sartor O, Witjes JA, Demkow T, et al. Multinational, double-blind, phase III study of prednisone and either satraplatin or placebo in patients with castrate-refractory prostate cancer progressing after prior chemotherapy: the SPARC trial. J Clin Oncol 2009; 27: 5431-8.

6 Kelly WK, Halabi S, Carducci MA, George DJ, Mahoney JF, et al. A 
randomized, double-blind, placebocontrolled phase III trial comparing docetaxel, prednisone, and placebo with docetaxel, prednisone, and bevacizumab in men with metastatic castration-resistant prostate cancer (mCRPC): Survival results of CALGB 9040. J Clin Oncol 2010; 28: 18 (suppl; abstr LBA4511).
7 Chi KN, Hotte SJ, Yu EY, Tu D, Eigl BJ, et al. Randomized phase II study of docetaxel and prednisone with or without OGX-011 in patients with metastatic castration-resistant prostate cancer. J Clin Oncol 2010 Aug 23. [Epub ahead of print].

8 James ND, Caty A, Borre M, Zonnenberg BA, Beuzeboc $\mathrm{P}$, et al.
Safety and efficacy of the specific endothelin-a receptor antagonist ZD4054 in patients with hormoneresistant prostate cancer and bone metastases who were pain free or mildly symptomatic: a double-blind, placebo-controlled, randomised, phase 2 trial. Eur Urol 2009; 55: 1112-23. 